\title{
Mental simulation inflates performance estimates for physical abilities
}

\author{
JOSHUA D. LANDAU \\ York College of Pennsylvania, York, Pennsylvania \\ TERRY M. LIBKUMAN \\ Central Michigan University, Mount Pleasant, Michigan \\ and \\ JONATHON C. WILDMAN, JR. \\ York College of Pennsylvania, York, Pennsylvania
}

\begin{abstract}
In five experiments, we examined how mental simulation of physical activities affected estimates of one's ability to perform the same activities. In Experiment 1, participants who simulated lifting a heavy object estimated that they could lift more weight than did participants who did not perform the simulation. In Experiment 2A, the frequency with which participants performed the simulation exercises was manipulated. In Experiments $2 \mathrm{~B}$ and 2C, we manipulated the amount of weight that people simulated lifting in order to address potential alternative explanations of the inflation effect. In Experiment 3, mental simulations were manipulated within subjects. In all the experiments, the simulated events showed inflated estimates, as compared with nonsimulated events. These results were interpreted in the context of the misattribution-of-familiarity account of imagination inflation.
\end{abstract}

The psychological literature is replete with examples of situations in which people possess mistaken beliefs about reality. For example, people will believe that they have generated a novel idea when they have inadvertently plagiarized another person's work, because they fail to recognize that someone else authored the idea (e.g., Landau \& Marsh, 1997; Marsh \& Landau, 1995). In other situations, people overestimate their own contributions to a joint product. Ross and Sicoly (1979) found that when married couples separately estimated their degree of responsibility for several activities (e.g., causing arguments, taking out the garbage, etc.), each couple's responses revealed an egocentric bias indicating that one or both of the spouses overestimated how much they contributed to the majority of the activities. Investigations of judgments of learning (JOL) indicate that people are often inaccurate in their predictions of future cognitive performance (e.g., Dunlosky \& Nelson, 1994; Nelson \& Dunlosky, 1991). In the JOL paradigm, people study a list of paired associates

Experiment 2 was presented at the 1999 meeting of the Eastern Psychological Association. Thanks are expressed to Chad C. Cooper, who assisted in the data collection for Experiment 2, and Richard L. Marsh and Andrew Leynes, who provided insightful comments on an earlier draft of the manuscript. Address correspondence to J. D. Landau, Department of Behavioral Sciences, York College of Pennsylvania, York, PA 17405-7199 or to T. M. Libkuman, Department of Psychology, Central Michigan University, Mt. Pleasant, MI 48859 (e-mail: jlandau@ ycp.edu or terry.m. libkuman@cmich.edu).

-Accepted by previous editorial team (e.g., TREE-DESK). Following each pair, people estimate their confidence that they will be able to remember the target word (DESK) when completing a cued-recall test later in the experimental session. When the JOL immediately follows learning the word pair, people's judgments are highly inaccurate for predicting future recall (i.e., high confidence does not result in accurate recall of the target words). Inadvertent plagiarism, egocentric bias, and inaccurate JOLs are just three experimental findings that highlight situations in which people's beliefs and reality are not synchronous.

When people engage in mental simulations, this seems to exaggerate the disparity between belief and reality. One of the more potent demonstrations of this phenomenon is imagination inflation (Garry, Manning, Loftus, \& Sherman, 1996; Goff \& Roediger, 1998; Heaps \& Nash, 1999; Hyman \& Billings, 1998; Paddock et al., 1998). In one such experiment, Garry et al. (1996) had people rate the likelihood that they had experienced certain childhood events (e.g., getting stuck in a tree, finding a $\$ 10$ bill, etc.). People returned to the laboratory 2 weeks later and mentally simulated some of the events from the first week. Afterward, people completed the rating task a second time. The rated likelihood of the simulated events that were originally assigned low ratings increased on the second questionnaire, indicating that mental simulation altered the participant's memories for childhood experiences. In contrast, the ratings of the nonsimulated events remained relatively unchanged. These results indicate that mentally simulating an event when it has not occurred can have deleterious effects on accurate recollections of the past. 
To date, imagination inflation research has been limited to investigations of how mental simulations affect memory for past events (i.e., people judge the likelihood that events have taken place sometime in the past). The question of how mental simulation affects predictions of future performance for physical abilities has not been addressed. This issue is important because many people use mental simulation to prepare themselves for future cognitive tasks. Taylor and her colleagues (e.g., Pham \& Taylor, 1999; Taylor, Pham, Rivkin, \& Armor, 1998) suggested that mental simulation improves performance by permitting people to envision alternative possibilities and eliminate potential errors. Mental simulation gives people the opportunity to gauge whether they are prepared to actually perform each part of the task. In this sense, mental simulation provides people with a way of estimating their current level of ability.

The main question that we examined is whether a brief mental simulation exercise would have a measurable impact on prospective estimates for physical abilities. Rather than focus on predictions of future cognitive abilities, we examined how mental simulations would influence predictions of physical performance. In the experiments that follow, participants mentally simulated physical activities and then estimated their proficiency at the activities. In Experiments 1-2C, the participants engaged in a mental simulation exercise in which they imagined lifting various amounts of weights and then estimated how much weight they could lift. In Experiment 1, half of the participants mentally simulated an event before making the estimation, and the other half did not. In the remaining experiments, several relevant variables were examined to determine their influence on the weight estimates. In Experiment 2A, we manipulated the frequency with which people performed the mental simulation exercise to ascertain whether it would affect their weight estimates (e.g., Goff \& Roediger, 1998). In Experiments 2B and 2C, we varied the amount of weight that the participants imagined lifting. Finally, to increase generality, in Experiment 3 we used a within-subjects design in which participants simulated several different physical events and then rated their abilities at these events.

\section{EXPERIMENT 1}

We hypothesized that mental simulation of an event would inflate performance prediction estimates for a physical ability. One group of participants mentally simulated lifting a heavy object (a refrigerator), whereas a second group did not perform the mental simulation. The participants then estimated how much weight they could lift. According to our literature review (e.g., Goff \& Roediger, 1998; Taylor et al., 1998), if mental simulation affects predictions about future performance, people in the mental simulation condition should provide higher weight estimates than do participants in the control condition. Alternatively, our mental simulation exercise, which was brief and lacking in detail, might not be potent enough to influ- ence performance predictions. It is also possible that people will discount the information from the mental simulation because they are aware of how much weight they are capable of lifting.

\section{Method}

Participants. Eighty-seven undergraduates were randomly assigned to either the no-simulation $(n=44)$ or the simulation $(n=$ 43) condition. Testing was conducted in small groups ranging from 5 to 10 participants.

Materials and Procedure. The participants in the simulation condition engaged in a mental simulation exercise in which the following instructions were read:

Close your eyes and imagine that you are helping a friend move and that it is your responsibility to move the refrigerator. Mentally picture yourself reaching around the appliance and gently lifting up and holding it there for a few seconds. Now, ease the refrigerator back to the ground and move away from it.

After completing the simulation exercise, which lasted approximately $15 \mathrm{sec}$, the participants reported the amount of weight they could benchpress. The participants in the no-simulation condition simply estimated how much weight they could benchpress.

\section{Results and Discussion}

Unless specified otherwise, the Type I error rate was set at .05 for all analyses reported in this paper. The purpose of this experiment was to determine whether a brief mental simulation exercise would have a measurable impact on performance estimates. As was predicted, those participants who mentally simulated lifting a heavy object reported being able to lift more weight $(M=114$ pounds, $S E=11.3)$ than did participants in the no-simulation condition $[M=88$ pounds, $S E=8.2 ; F(1,84)=3.4, p<.05] .^{1}$ We believe that the weight estimates increased because the mental simulation exercise made it easier for people to believe that they could lift more weight. Goff and Roediger (1998) speculated that imagination inflation for retrospective memories results from a confluence of factors. They argued that simulating an event increases its familiarity, which makes it easier to bring the event to mind. Later, when making judgments about the event, people mistake the fluency with which the idea comes to mind for evidence that the event actually occurred in the past. People misinterpret the increased familiarity as an indication of a real event, and not of a simulated event (e.g., Johnson, 1988; Johnson, Raye, Hasher, \& Chromiak, 1979). There is a substantial body of evidence suggesting that people do not sufficiently monitor the source of the familiarity when making judgments such as confidence ratings and task difficulty (Jacoby \& Kelley, 1987; Johnson, Hashtroudi, \& Lindsay, 1993; Kelley \& Jacoby, 1996; Kelley \& Lindsay, 1993; Marsh, Landau, \& Hicks, 1997). Consequently, when judging the occurrence of a simulated event, people confuse the source of the familiarity and incorrectly claim that the event was actually experienced, when, in fact, it was not. In a similar fashion, when people made their weight estimates after the refrigerator simulation exercise, the details of the mental simulation exercise came to mind easily. Rather than discounting this information as the residue from the simulation exercise, people used 
it as a basis for their judgments. Consequently, they reported inflated weight estimates.

Although the misattribution account is plausible, there are several alternative explanations to consider. First, it is possible that the participants assigned to the simulation condition could actually lift more weight than those assigned to the no-simulation condition. This explanation is unlikely, because we randomly assigned participants to the two conditions. Second, it is possible that the people in the simulation condition provided higher weight estimates because of demand characteristics. That is, they believed that the experimenter desired a higher estimate, and they inflated their estimates in an effort to help the experimenter. Previous literature, however, suggests that demand characteristics are not a viable explanation. Gregory, Cialdini, and Carpenter (1982) reported that imagery inflation was not due to demand characteristics. In their experiment, people imagined being arrested for petty theft and shoplifting. People in a control group read neutral scenarios that were not crime related. The researchers contacted the people later that evening as part of a seemingly unrelated telemarketing survey. Each person answered a series of questions, including an estimate of how likely it was that they would be arrested for different crimes. People in the imagination condition thought that it was more likely that they could be arrested for petty theft or shoplifting. Importantly, none of the people claimed that they were aware of the relationship between the experimental session and the survey.

Gregory et al.'s (1982) results indicate that people will inflate their estimates even when the potential contribution of demand characteristics is small. The previous literature notwithstanding, we designed the following experiments, in part, to investigate the demand characteristic explanation more fully. In Experiments $2 \mathrm{~A}, 2 \mathrm{~B}$, and $2 \mathrm{C}$ we used a disguised paradigm that was conceptually similar to the one used by Gregory et al. Disguising the weight estimate as part of a separate, unrelated study should decrease the probability that people intentionally inflate their weight estimates in an effort to help the experimenter.

\section{EXPERIMENTS 2A-2C}

The motivation for Experiments $2 \mathrm{~A}-2 \mathrm{C}$ was to address the possibility that demand characteristics played a role in producing the effect reported in Experiment 1 and to manipulate variables that might affect the amount of weight that people would claim to be able to lift. We attempted to reduce demand characteristics by leading the participants to believe that they would take part in two separate research projects, whereas in truth, the two projects were related. The people first simulated lifting weights, and then they reported aspects of their imagery experience. The second study was a health survey. This survey contained one critical item that asked the participants to estimate how much weight they thought they could lift. If demand characteristics are the sole explanation of the inflated estimates in Experiment 1, disguising the relationship between the imagery questionnaire and the health survey should reduce the likelihood that the people would intentionally inflate their weight estimates. In order for the people to intentionally inflate their weight estimates, they would first have to discover the relationship and then decide to add weight to their estimates as a way to appease the experimenter.

If inflation of weight estimates and imagination inflation (Garry et al., 1996; Goff \& Roediger, 1998) are produced by the same mechanism, manipulations that influence imagination inflation should have similar effects on weight estimates. In their investigation of imagination inflation, Goff and Roediger had participants either perform or imagine simple activities (e.g., breaking a toothpick in your mouth, tugging your earlobe, etc.) one, three, or five times. The participants then decided whether they had actually performed a list of activities. Some of these activities had been performed earlier, some had only been imagined, and some were new activities that were neither performed nor imagined. As the number of imagination trials increased, people came to believe they had actually performed an activity, when, in fact, they had not. On the basis of this finding, we manipulated the number of times that people mentally simulated lifting 200 pounds $(0,5$, or 10 simulation trials) in Experiment $2 \mathrm{~A}$. We hypothesized that increasing the number of simulation trials would increase weight estimates. Moreover, if demand characteristics were operating in this experiment, the people performing 10 simulations would have to intentionally decide to add more weight than would the people performing 5 simulations.

The primary motivation for Experiment $2 \mathrm{~B}$ was to determine whether the magnitude of the weight mentioned in the mental simulation exercise would affect prospective weight estimates. Other researchers have found that the details of the mental simulation exercise influence performance (e.g., Intons-Peterson \& Roskos-Ewoldsen, 1989; Taylor et al., 1998). For example, Intons-Peterson and Roskos-Ewoldsen asked people to mentally travel specified routes and to press a button when they arrived at the destination point. During this mental trip, people imagined transporting objects of increasing weight (e.g., a balloon, a ball, and a cannonball). As the weight of the objects increased, so did the mental transport times. Thus, the actual weight of each object in the mental simulation exercise played an important role in determining how quickly people traveled mentally. To investigate how the amount of weight people imagined lifting would affect their estimates, some of our participants imagined lifting 20 pounds, whereas other participants imagined lifting a more substantial amount of weight (200 pounds) or no weight at all (a control condition). A secondary motivation for this experiment was to further investigate the demand characteristics account. It is possible that performing any mental simulation, regardless of the details, causes an inflation of performance estimates. If this is true, people who engage in simulations that differ in magnitude should provide similar weight estimates. If, however, the amount of weight that people simulate has an influence on their weight estimates, 
people who imagine lifting a great deal of weight should provide higher weight estimates than do people who simulate lifting less weight.

We conducted Experiment $2 \mathrm{C}$ to determine the extent to which people use the amount of simulated weight as a reference point for their estimates. ${ }^{2}$ By this account, a misattribution of familiarity does not influence performance estimates. Rather, people simply use the amount of weight in the simulation exercise to anchor their estimates. Participants who simulate lifting 200 pounds may start at 200 pounds and then shift their estimates downward to a more manageable weight. Likewise, participants who simulate 20 pounds may shift upward to a more realistic weight. If this account is accurate, people who simulate a larger amount of weight should always report that they can lift more weight. To evaluate this anchoring hypothesis, we compared the weight estimates from a group who imagined lifting 200 pounds with those from a group who imagined lifting 400 pounds. If people are anchoring their estimates, people in the 400-pounds condition should start with a higher value before adjusting to a more realistic weight estimate. Because they start with a higher value, it is likely that the adjustment process will produce a higher weight estimate. Therefore, if anchoring is a viable explanation, people in the 400-pounds condition should provide a higher weight estimate than do people in the 200 pounds condition.

\section{Method}

Participants. In Experiment 2A, 82 undergraduates were randomly assigned to perform 0 simulations $(n=27), 5$ simulations $(n=22)$, or 10 simulations $(n=33)$. In Experiment 2B, 129 undergraduates were assigned to the control $(n=44), 20$-pounds $(n=$ $45)$, or 200-pounds $(n=40)$ condition. ${ }^{3}$ In Experiment 2C, 119 undergraduates were randomly assigned to the 200-pound $(n=36)$, 400 -pounds $(n=36)$, or control $(n=47)$ condition. In all three experiments, the participants were tested in small groups of $8-10$, and none of them had taken part in Experiment 1.

Materials and Procedure. At the outset of the experimental session, the participants in the simulation conditions were informed that they would take part in two separate studies. The first study was an investigation of how people mentally simulate an event, and the second study consisted of a health survey. In order to increase the effectiveness of the cover story, one experimenter conducted the mental simulation study, and a second experimenter administered the health survey.

In Experiment 2A, the participants in the 5- and 10-simulation conditions listened to the following minute-long audiotaped instructions:

Close your eyes, feel confident that you will be able to lift the 200 pound barbells, mentally picture yourself lying on the gray weight bench with the bar above your head, concentrate on lifting the bar while blocking out other sights and sounds, take a look at the two 100-pound weight plates, grab the bar, take a deep breath and gently lift it off the stand, gradually thrust it in the air and breathe out, return the bar to the stand and get up from the bench.

The mental simulation instructions were repeated 5 or 10 times, with a short rest interval between each repetition. The participants then completed the 12-item survey that assessed the qualitative aspects of their simulation experience and further supported the notion that this was an investigation of their mental simulation experience. For instance, people were asked the following questions. What color were the weights? Was there someone spotting you? Was there a sweaty smell in the gym? Was it warm in the gym? Were you breathing heavily after returning the bar to the bench? In addition to these questions, the participants also rated the vividness of their mental simulation experience on a scale ranging from 1 to 10 (1, extremely poor; 10 , extremely good).

The participants in the 5- and 10-simulation conditions then completed a 16-item survey that asked them to provide some basic demographic information (e.g., age, gender), as well as some health related information (Do you smoke? Do you exercise on a regular basis? Compared with the average student, do you think that you are less healthy or more healthy? How many times have you visited the doctor this year?). The critical survey item, which was the seventh question on the survey, asked the participants to estimate how much weight they thought they could lift. People were allowed $5 \mathrm{~min}$ to complete each survey. Following completion of the health survey, the experimenter asked the participants in the 5- and 10-simulation conditions whether they noticed the relationship between the two studies and to describe the exact nature of this relationship. The people in the 0 -simulation condition did not experience any simulation, and therefore these participants only completed the health survey.

The participants in Experiment 2B listened to the mental simulation exercise two times, completed the imagery survey, and then completed the health survey. The participants in the 20-pounds condition mentally simulated lifting 20 pounds, whereas the participants in the 200-pounds condition simulated lifting 200 pounds. Other than the change in weight, these two conditions were identical. The participants in the control condition did not perform a mental simulation exercise and, therefore, only filled out the health survey.

In Experiment 2C, we used the same procedures and materials as those used in Experiment 2B, except that the participants in the 200pounds condition mentally simulated lifting 200 pounds, whereas the participants in the 400-pounds condition simulated lifting 400 pounds. As with the other control conditions, these participants did not imagine lifting any weight.

\section{Results and Discussion}

Experiment 2A. None of the participants in the 5- and 10 -simulation conditions reported detecting a relationship between the imagery and the health survey studies. It is therefore unlikely that these people intentionally inflated their weight estimates on the health survey. The participants in the 0 -simulations condition estimated that they could lift 88 pounds $(S E=9.09)$, the participants in the 5simulations condition estimated 111 pounds $(S E=17.16)$, and the participants in the 10-simulation condition averaged 132 pounds $(S E=13.4)$. The overall analysis of variance (ANOVA) fell just shy of conventional statistical significance $[F(2,79)=2.8, p=.06]$. However, a significant linear trend was present $[F(1,79)=5.7, p<.02]$. Those participants who completed more mental simulation trials believed that they could lift more weight than did the participants who completed fewer simulations. These results fit nicely with the misattribution account. In this case, increasing the number of simulation trials causes the details of the simulation to come to mind more easily, which in turn causes people to inflate their weight estimates. These results are consistent with Goff and Roediger (1998), who reported that the number of memory distortions increased as the number of imaginings increased.

At least two additional aspects of these data are particularly noteworthy. First, if demand characteristics were a 
viable explanation of the estimation inflation found in Experiment 1 , people in the 10-simulation condition would have to be aware that another condition existed, and then they would have to intentionally increase their estimates. However, none of the participants in either of the simulation conditions noticed the relationship between the two studies, and because we used a between-subjects design, it is unlikely that they knew that other people were performing the simulation only five times. Therefore, it is unlikely that the people in the 10-simulationscondition consciously inflated their estimates. In our view, the participants believed that the weight estimates reflected the amount of weight they were capable of lifting. Second, the participants in the 0 -simulation condition provided the same weight estimate as did the participants in the control group of Experiment 1 (88 pounds). Because neither group engaged in mental simulation, these data provide convergent evidence for baseline performance in this population.

Experiment 2B. As in Experiment 2A, none of the participants recognized the relationship between the two studies. The weight that the participants mentally simulated did influence their weight estimates $[F(2,126)=5.63, p<$ $.05]$. Those participants who did not perform the mental simulation predicted that they could lift 83 pounds $(S E=$ 7.25 ), whereas the participants in the 20-pounds condition predicted 61 pounds $(S E=7.28)$ and the participants in the 200-pounds condition predicted 97 pounds ( $S E=$ 8.42). Planned comparisons revealed that the participants in the 20-pounds condition reported they could lift less weight than did the participants in the control condition $[t(87)=2.14, p<.05]$ and the participants in the 200pounds condition $[t(83)=3.24, p<.05]$. Although the participants in the 200-pounds condition estimated that they could lift more weight than the participants in the control condition (14 pounds), this result was not significant $[t(82)=1.25, p>.05]$. Consistent with the misattribution account, the detail of the mental simulation exercise has predictable effects over the subsequent weight estimates. Because most people do not know exactly how much weight they can lift, the content of the simulation exercise serves as a source of information for people to use when making their weight estimates. Therefore, it is not surprising to find that the mental simulation exercise can either increase (Experiments 1 and 2A) or decrease performance estimates (Experiment 2B). The results of this experiment indicate that when the participants mentally simulated lifting a small amount of weight, they provided lower weight estimates than did the participants in a control condition or the participants who simulated a large amount of weight.

Experiment 2C. As with the two previous experiments, none of the participants in the experimental conditions recognized the relationship between the imagery exercise and the health survey. The participants in the control condition estimated that they could lift 82 pounds $(S E=8.56)$, whereas the people in the 200-pounds $(M=$ $114, S E=8.9)$ and 400 -pounds $(M=117, S E=11.4)$ conditions estimated that they could lift substantially more weight $[F(2,116)=4.32, p<.05]$. Planned com- parisons indicated that the 200- and the 400-pounds groups were similar $(t<1)$ but that they both differed from the control group (both $t \mathrm{~s}>2.36$ ). If the participants were blindly using the amount of weight as a reference point with which to anchor their predictions, the participants in the 400-pounds condition should have provided a higher weight estimate. The fact that the people did not do this shows that they assess the plausibility of the contents of the mental simulation and that this plays some role in how people use that information to make their estimates.

\section{EXPERIMENT 3}

In Experiment 3, we addressed the generality of the effect reported in the previous four experiments by using an experimental design modeled after Garry et al.'s (1996) imagination inflation paradigm. The purpose of this experiment was twofold. First, we wanted to determine whether people would alter their performance estimates after engaging in a mental simulation exercise. We used a withinsubjects design that allowed us to measure people's beliefs about their abilities before and after the mental simulation exercises. Second, we examined whether mental simulation would have the same enhancing effects for behaviors other than the weightlifting estimation used in the four previous experiments. We had the participants complete a 10item questionnaire that assessed their abilities for several sports-related activities (e.g., shooting free throws, throwing darts, etc.). The participants returned after a 1-week delay and mentally simulated half of the activities before completing the questionnaire for a second time. We predicted that the performance estimates for the simulated activities would increase and those for the nonsimulated activities would remain stable. A significant interaction between the test (pretest or posttest) and the item type (simulated or not simulated) was expected.

\section{Method}

Participants, Materials, and Procedure. Twenty-three undergraduates received extra credit in exchange for their participation. As in the previous experiments, we tested all the participants in small groups, and none had participated in the four previous experiments.

During the first session, we informed the participants that we were interested in their abilities at certain athletic activities. We asked them to complete a 10-item questionnaire (see the top half of Table 1 for selected questions). They were encouraged to answer each question to the best of their ability, making the best estimate possible if they had never performed one of the activities or were otherwise unsure about their expected performance. None of the participants said that they were unfamiliar with the activities.

The participants returned for the second experimental session following a 1-week delay. The 10 questionnaire items were randomly divided into two subsets, each containing five activities. The participants were randomly assigned to one of the two subsets. They were asked to mentally simulate the five critical activities in that subset, whereas the other activities served as a control. For example, one group of participants simulated the five activities in Subset A (walking, putting [golf], throwing a baseball [speed], soccer goalie [number of blocks], and bench pressing weights) and did not simulate the five activities in Subset B (shooting free throws, throwing a football [distance], kicking an extra point, throwing darts [number of bull's-eyes], 
Table 1

Selected Questions From the Sports Questionnaire and a Sample Imagery Exercise Used in Experiment 3

If you were to shoot 10 free throws on a regulation basketball court, how many would go in?

$0-1-2-3-4-5-6-7-8-9-10$ baskets

How far can you throw a football?

$0-5-10-15-20-25-30-35-40-45-50-55-60-65-70-75+$ yards

How high is your vertical leap?

$0-6-12-18-24-30-36-42-48-54-60-66-72$ inches

\section{Putting}

close your eyes

feel confident that you will be able to make the putt

mentally picture yourself with both feet flat on the green grass

see the hole as being very large

concentrate on swinging the club straight back and hitting the ball

solidly without other sights and sounds

imagine that the ball travels directly to the hole and plops into the cup feel very confident and happy that the ball went into the cup

What color was your club?

Was there a flagpole sticking out of the hole?

Were you nervous that the ball might miss the cup?

and vertical leap). A second group of participants simulated the five activities in Subset B but did not simulate the five activities in Subset A.

The participants were informed that they would complete the five mental simulation exercises that were described in their experimental packets. Each description contained a series of steps for the simulation exercise (see the bottom half of Table 1 for an example). Below each described activity, there were three questions about what they had simulated. These questions were designed to ensure that the participants complied with the task instructions.

In an effort to give people practice with mentally simulating an event, instructions for a sample mental simulation exercise were read aloud:

Close your eyes; mentally picture yourself standing in the doorway of your home. As you enter the house take a look around and begin counting the windows. Slowly make your way to the different rooms and count the windows in each room. After visiting all of the rooms, return to the doorway and exit the house.

Following the practice exercise, the participants wrote their answers to the following questions regarding their experience. How many windows do you count? Was it sunny outside? What was the temperature inside the house?

The participants had $60 \mathrm{sec}$ to complete each simulation exercise. The participants then had $30 \mathrm{sec}$ to write answers to the three questions. This procedure was repeated for the five target activities. After all five exercises were completed, the experimenter announced that the sports activities questionnaires that they completed during the first week had been accidentally misplaced, and the participants were then asked to complete the questionnaire a second time. Following administration of this posttest, we debriefed and dismissed all of the participants from the experimental session.

\section{Results and Discussion}

We converted participant responses to proportions based on the highest level of performance for the simulated and not-simulated questionnaire items (see Table 2). For example, for the free throw question, we divided each response by 10 , and for the vertical leap question, we divided each response by 72 . These proportions were then averaged and analyzed with a 2 (test, pretest vs. posttest) $\times 2$ (item type, simulated vs. not simulated) repeated measures ANOVA. Overall, the performance estimates increased over the 1week delay $[F(1,22)=11.28, p<.05]$. The item type main effect did not reach statistical significance $[F(1,22)=1.55$, $p=.22]$. The test $\times$ item type interaction was significant $[F(1,22)=4.73, p<.05]$. Post hoc analyses indicated that the performance estimates for the simulated activities increased $[t(22)=3.82, p<.05]$, whereas the estimates for the not-simulated activities did not increase $[t(22)=$ $1.54, p>.05]$.

It is possible that we failed to detect a significantincrease for the not-simulated events because of a Type II error. To address this concern, we performed a power analysis on both post hoc comparisons. The power values for the simulated and not-simulated activities were .954 and .314 , respectively, and the effect sizes were .399 and .097. Although it is possible that the not-simulated activities increased during the intervening week and that we are not detecting this effect because of a power problem, the data suggest that this increase is quite small. Thus, we would need hundreds of participants to increase power in order to detect such a small effect. We believe that the interaction term demonstrates that the simulated activities increased more than did the not-simulated activities. Simulating an event has some effect on people's estimates of their future performance. The other point that we wish to make is that our results are consistent with the existing imagination inflation literature in that the imagined events showed a larger increase than the not-imagined items (e.g., Garry, Sharman, Wade, Hunt, \& Smith, 2001; Heaps \& Nash, 1999; Pezdek \& Eddy, 2001). In the imagination inflation literature, the not-imagined items tended to increase, but this increase was typically smaller than the increase associated with the imagined items.

The results from this experiment indicate that mentally simulating an activity increased performance estimates. This finding is consistent with the results from the betweensubjects designs (i.e., Experiments 1-2C). The results from Experiment 3 are also meaningful, because they help to rule out demand characteristics as a viable explanation for these effects. In order for demand characteristics to fully explain these results, our participants would have needed to remember their exact ratings for all 10 activities from the first week. Then, during the second week, they would have to boost their ratings for the simulated activities while keeping the ratings for the other activities consistent. Because it is unlikely that people remembered the

Table 2

Mean Performance Ratings (With Standard Error) for Experiment 3

\begin{tabular}{clllll}
\hline & \multicolumn{3}{c}{ Session } \\
\cline { 2 - 3 } \cline { 5 - 6 } Item Type & \multicolumn{2}{c}{ Week One } & & \multicolumn{2}{c}{ Week Two } \\
\cline { 2 - 6 } \cline { 2 - 6 } & $M$ & $S E$ & & $M$ & $S E$ \\
\hline Not-simulated & .31 & .02 & & .34 & .02 \\
Simulated & .33 & .03 & & .39 & .03 \\
\hline
\end{tabular}


exact rating for each of the activities, we doubt that demand characteristics provide a complete explanation of our results. If demand characteristics had inflated the weight estimates, only the main effect for session, and not the two-way interaction, would have been significant.

\section{GENERAL DISCUSSION}

The purpose of this research was to determine whether a brief mental simulation exercise influences prospective estimates for physical abilities. After people imagined themselves lifting a heavy object, they claimed that they could lift more weight than did people who had not engaged in a mental simulation exercise (Experiment 1). Increasing the frequency of the simulation exercise resulted in higher estimates (Experiment 2A). People who simulated lifting a larger amount of weight estimated that they could lift more weight than did people who simulated lifting a smaller amount of weight (Experiment 2B). Importantly, we demonstrated that anchoring was not a viable explanation of estimation inflation, because people who mentally simulated 200 or 400 pounds provided similar weight estimates (Experiment 2C). Finally, people increased their performance estimates for activities that they mentally simulated, whereas activities that people did not simulate remained relatively stable (Experiment 3 ).

Several aspects of the results from these experiments raise serious doubts that the inflated weight estimates were a result of demand characteristics. First, Experiments $2 \mathrm{~A}-$ $2 \mathrm{C}$ used a procedure that disguised the relationship between the mental simulation exercise and the weight estimate, and none of the participants in these experiments reported that they were aware of the relationship between the two studies. Second, if demand characteristics were an accurate explanation of these results, the participants in the different experimental conditions should have provided roughly the same weight estimates. For instance, the results from Experiment 2B demonstrate that the participants in the 200-pounds condition predicted that they could lift more weight than did people in the 20-pounds condition. It is unclear why the participants in the 200-pounds condition would be compelled to assist the experimenter more than would people in the 20-pounds condition. Third, mental simulations affected weight estimates in betweensubjects designs (Experiments 2A-2C) and a withinsubjects design (Experiment 3 ).

The results from the present experiments demonstrate that even a brief and impoverished mental simulation exercise is a powerful tool that can influence people's ability to predict future physical performance. Although our paradigm required prospective performance estimates, rather than recollections of past experiences, we suspect that the same cognitive processes produce imagination-induced distortions for both types of judgments. In both cases, people's judgments of an ambiguous situation are influenced by the most available information. In the present case, people failed to consider that their weight estimates were influenced by the simulation exercise, and consequently, they offered higher (Experiment 2C) or lower (Experiment 2B) estimates for the imagined tasks. The results from Experiment $2 \mathrm{C}$ are interesting because they show that people do not blindly anchor their weight estimates to the values mentioned in the mental simulation exercise. Like other misattribution-of-familiarityeffects, people appear to have some cognitive control over this error (e.g., Whittlesea, Jacoby, \& Girard, 1990). A similar misattribution of availability might cause the inaccuracy of the immediate JOLs (cf. Nelson \& Dunlosky, 1991). Specifically, when people attempt to immediately predict future memory performance for a target word, they tend to rely on the availability of the word to make their judgment. Because the study pair still resides in short-term memory, this interferes with people's ability to accurately predict future recall of the target word. The common thread in these two cases is that information that easily comes to mind influenced subsequent judgments.

In terms of future research, we suspect that several factors will influence the magnitude of the estimation inflation. For example, if the simulation exercise is concrete and incorporates tangible details, the weight estimates should increase. People who imagine realistic and/or plausible events should offer performance estimates that outweigh those made by people who imagine less realistic events (e.g., Pezdek, Finger, \& Hodge, 1997).

Mental simulation is a powerful technique that benefits mnemonic (Yesavage, Rose, \& Bower, 1983) and athletic (Wrisberg \& Anshel, 1989) performance. However, research indicates that mental simulation exercises can yield unintended side effects. Mentally simulating that an event has taken place, when it has not, causes people to believe that the event actually occurred (Garry et al., 1996). Simulations also have the ability to influence attitudes and behavior (Gregory et al., 1982). Our results are important because they provide evidence that physical ability judgments are constructed much like our recollections of the past and that they are similarly malleable following a brief mental simulation exercise. Thus, people who use mental simulation exercises to monitor their performance should be careful to make sure that they are not over- or underestimating their actual performance level.

\section{REFERENCES}

Dunlosky J., \& Nelson, T. O. (1994). Does sensitivity of judgments of learning (JOLs) to the effects of various study activities depend on when the JOLs occur? Journal of Memory \& Language, 33, 545-565.

Garry, M., Manning, C. G., Loftus, E. F., \& Sherman, S. J. (1996). Imagination inflation: Imagining a childhood event inflates confidence that it occurred. Psychonomic Bulletin \& Review, 3, 208-214.

Garry, M., Sharman, S. J., Wade, K. A., Hunt, M. J., \& Smith, P. J. (2001). Imagination inflation is a fact, not an artifact: A reply to Pezdek and Eddy. Memory \& Cognition, 29, 719-729.

Goff, L. M., \& RoEDIGER, H. L., III (1998). Imagination inflation for action events: Repeated imaginings lead to illusory recollections. $\mathrm{Mem}$ ory \& Cognition, 26, 20-33.

Gregory, W. L., Cialdini, R. B., \& Carpenter, K. M. (1982). Selfrelevant scenarios as mediators of likelihood estimates and compliance: Does imagining make it so? Journal of Personality \& Social Psychology, 43, 89-99. 
HEAPS, C., \& NASH, M. (1999). Individual differences in imagination inflation. Psychonomic Bulletin \& Review, 6, 313-318.

Hy man, I. E., JR., \& Billings, F. J. (1998). Individual differences and the creation of false childhood memories. Memory, 6, 1-20.

Intons-Peterson, M. J., \& Roskos-Ewoldsen, B. B. (1989). Sensoryperceptual qualities of images. Journal of Experimental Psychology: Learning, Memory, \& Cognition, 15, 188-199.

JACOBY, L. L., \& KeLLEY, C. M. (1987). Unconscious influences of memory for a prior event. Personality \& Social Psychology Bulletin, 13, 314-336.

Johnson, M. K. (1988). Reality monitoring: An experimental phenomenological approach. Journal of Experimental Psychology: General, 117, 390-394.

Johnson, M. K., Hashtroudi, S., \& LindSAY, D. S. (1993). Source monitoring. Psychological Bulletin, 114, 3-28.

Johnson. M. K., Raye, C. L., Hasher, L., \& Chromiak, W. (1979). Are there developmental differences in reality-monitoring? Journal of Experimental Child Psychology, 27, 120-128.

Kelley, C. M., \& JACOBY,L. L. (1996). Adult egocentrism: Subjective experience versus analytic bases for judgment. Journal of Memory \& Language, 35, 157-175.

Kelley, C. M., \& Lindsay, D. S. (1993). Remembering mistaken for knowing: Ease of retrieval as a basis for confidence in answers to general knowledge questions. Journal of Memory \& Language, 32, 1-24

LANDAU, J. D., \& MARSH, R. L. (1997). Monitoring source in an unconscious plagiarism paradigm. Psychonomic Bulletin \& Review, 4, 265 270

MARSH, R. L., \& LANDAU, J. D. (1995) Item availability in cryptomnesia: Assessing its role in two paradigms of unconscious plagiarism. Jour nal of Experimental Psychology: Learning, Memory, \& Cognition, 21, $1568-1582$

Marsh, R. L., Landau, J. D., \& Hicks, J. L. (1997). The contribution of inadequate source monitoring to unconscious plagiarism during idea generation. Journal of Experimental Psychology: Learning, Memory, \& Cognition, 23, 886-897.

Nelson, T. O., \& Dunlosky, J. (1991). When people's judgments of learning (JOLs) are extremely accurate at predicting subsequent recall: The "delayed-JOL effect." Psychological Science, 2, 267-270.

Paddock, J. R, Joseph, A. L., Chan, F. M., Terranova,S., Manning, C., \& LoFTUS, E. F. (1998). When guided visualization procedures may backfire: Imagination inflation and predicting individual differences in suggestibility. Applied Cognitive Psychology, 12, 63-75.
Pezdek, K., \& EdDy, R. M. (2001). Imagination inflation: A statistical artifact of regression toward the mean. Memory \& Cognition, 29, 707-717.

Pezdek, K., Finger, K., \& Hodge, D. (1997). Planting false childhood memories: The role of event plausibility. Psychological Science, $\mathbf{8}$, 437-441.

Pham, L. B., \& TAY LOR, S. E. (1999). From thought to action: Effects of process- versus outcome-based mental simulations on performance. Personality \& Social Psychology Bulletin, 25, 250-260.

Ross, M., \& Sicoly, F. (1979). Egocentric biases in availability and attribution. Journal of Personality \& Social Psychology, 37, 322-336.

Taylor, S. E., Pham, L. B., Rivkin, I. D., \& Armor, D. A. (1998). Harnessing the imagination: Mental simulation, self-regulation, and coping. American Psychologist, 53, 429-439.

Whittlesea, B. W. A., JACOBy, L. L., \& Girard, K. (1990). Illusions of immediate memory: Evidence of an attributional basis for feelings of familiarity and perceptual quality. Journal of Memory \& Language, 29, 716-732.

Wrisberg, C. A., \& ANSHEL, M. H. (1989). The effects of cognitive strategies on the free throw shooting performance of young athletes. Sport Psychologist, 3, 95-104.

Yesavage, J. A., Rose, T. L., \& Bower, G. H. (1983). Interactive imagery and affective judgments improve face-name learning in the elderly. Journal of Gerontology, 38, 197-203.

\section{NOTES}

1. We removed one statistical outlier from the data analysis, leaving 43 participants in both conditions. Because our hypothesis was directional, we report one-tailed significance tests in this experiment.

2. We would like to thank Deanne Westerman for raising this potential explanation.

3. Following a reviewer's suggestion, we added a control group to this experiment after we collected the data from the 20-pound and 200-pound groups. To ensure some type of random assignment, we also added participants to the 20- and 200-pound conditions. It is important to note that the additional data did not substantially alter the means or standard errors for these two groups.

(Manuscript received March 18, 1999; revision accepted for publication January 9, 2002.) 